\title{
Spatial analysis of Neospora caninum distribution in dairy cattle from Sweden
}

\author{
Jenny Frössling ${ }^{1}$, Ane Nødtvedt ${ }^{2}$, Ann Lindberg ${ }^{1,3}$, Camilla Björkman² \\ ${ }^{1}$ Department of Disease Control and Epidemiology, National Veterinary Institute, SE-751 89 Uppsala, \\ Sweden; ${ }^{2}$ Department of Clinical Sciences, Swedish University of Agricultural Sciences, P.O. Box 7054, SE- \\ 75007 Uppsala, Sweden; ${ }^{3}$ Swedish Dairy Association, P.O. Box 210, SE-101 24 Stockholm, Sweden
}

\begin{abstract}
The national herd prevalence and spatial distribution of Neospora caninum infected dairy herds in Sweden were investigated. The study was based on a bulk milk survey comprising samples from 2,978 herds. Test-positive herds were found in all parts of Sweden and the overall prevalence of test-positive herds was $8.3 \%(95 \%$ confidence interval $=7 \cdot 3-9.3 \%)$. The presence of spatial autocorrelation was tested using the Moran's $I$ test. Possible clusters of test-positive herds were identified by applying the local indicator of spatial association (LISA) test statistic and the spatial scan statistic. Analysis based on data aggregated by postal code areas as well as analysis based on exact coordinates identified significant clusters of high prevalence in the middle parts of Sweden and low prevalence in the south. This was not expected considering the results from other European studies of N. caninum in cattle. However, the findings are supported by the distribution of previously known case herds.
\end{abstract}

Keywords: cluster analysis, prevalence, Neospora caninum, cattle, bulk milk.

\section{Introduction}

The protozoan parasite Neospora caninum is of significance mainly because of its ability to cause abortion and persistent infection in cattle. Although the full nature of the parasite-host interaction is still unclear, it is known that infection can be vertical, i.e. by transmission of tachyzoites from cow to offspring during pregnancy, or horizontal, by ingestion of oocysts shed by a definitive host (Dubey et al., 2007). So far, identified definitive hosts include the dog and the coyote (McAllister et al., 1998; Gondim et al., 2004) and there are other potential host species within the Canidae family.

Corresponding author:

Jenny Frössling

Department of Disease Control and Epidemiology

National Veterinary Institute (SVA)

SE-751 89 Uppsala, Sweden

Tel. +461867 4359; Fax +4618674445

E-mail: jenny.frossling@sva.se
The presence of N. caninum antibodies has been demonstrated in cattle populations worldwide but the estimated prevalence of cattle infected with $N$. caninum varies considerably between herds, regions and countries. In a European study, the herd prevalence in the southern and middle parts of Sweden was estimated to $16 \%$, whereas $49 \%, 63 \%$ and $76 \%$ of investigated dairy herds in Germany, Spain and The Netherlands had at least one seropositive cow (Bartels et al., 2006). The reason for the regional differences is only partially known, but dog density and climatic factors have been suggested as explanations (Schares et al., 2003; Rinaldi et al., 2005). The presence of coyotes has also been found to be a risk factor (Barling et al., 2000). In Sweden, serological surveys have shown that the prevalence of N. caninum is $0.5 \%$ in dogs (Björkman et al., 1994) and 2\% in individual dairy cattle (Björkman et al., 2000). Bulk milk screenings of herds in the southern and middle parts of Sweden estimate the herd prevalence in these areas at approximately $20 \%$ (unpublished data). 
The aim of this study was to estimate the national prevalence and the general spatial distribution of $N$. caninum infected Swedish dairy herds based on bulk milk testing. As this is the first N. caninum survey of its kind in Sweden we found it of interest to look for possible geographical patterns, as well as the presence of specific infection clusters. We also used geographical tools to investigate whether the study sample was representative of the target population as this was considered essential for the interpretation of results.

\section{Materials and methods}

\section{Study sample}

The study material was selected from bulk milk samples that were collected in 2002 and submitted for analysis within the mandatory surveillance programmes for Enzootic Bovine Leucosis and Infectious Bovine Rhinotracheitis. Sweden is free from these diseases and in 2002 surveillance activities included the collection of bulk milk samples from all milk-delivering herds (Anonymous, 2008). The study sample consisted of specimen from 2,978 herds, corresponding to approximately $30 \%$ of all dairy herds present in the country that year. Samples were selected at the laboratory by convenience sampling, i.e. not through a strictly random procedure, and not under control by the investigators.

\section{Diagnostic test}

An iscom enzyme-linked immunosorbent assay (iscom ELISA) was used to demonstrate the presence of specific antibodies to N. caninum (Björkman et al., 1997; Frössling et al., 2003). When applied to bulk milk samples from Swedish dairy herds, this test has been estimated to have a diagnostic sensitivity of $\sim 50 \%$ (95\% confidence interval $(\mathrm{CI})=21$ $79 \%)$ and a specificity of $\sim 81 \% \quad(95 \% \mathrm{CI}=72-$ $89 \%$ ) (Frössling et al., 2006). These estimates refer to the ability to detect two or more test-positive cows within a herd.

\section{Location data}

The locations of herds in the study sample and all dairy herds in Sweden were specified by three digit postal codes $(n=276)$ and exact coordinates. The total area of Sweden is $449,964 \mathrm{~km}^{2}$ (Anonymous, 2001) and the median size of postal code areas is approximately $284 \mathrm{~km}^{2}$ (boundaries of postal code areas are shown in Figure 1). Postal codes and coordinates were retrieved from the databases of the Swedish Dairy Association (study sample = 2002; background population $=2006$ ) and of the Swedish Board of Agriculture (2006), respectively. The geographical distribution of herds and possible clustering were investigated by utilizing both data aggregated by postal code area and point data. Applicable postal codes and coordinates were available for 2,944 and 2,580 of the 2,978 herds in the study sample, respectively. In the background population of 2006, postal codes were available for all herds. The herd density of the background population per postal code area is presented in Figure 1.

\section{Spatial analysis}

The $N$. caninum prevalence was defined as the proportion of test-positive herds of the total number of sampled herds and was calculated for each postal code area. Due to differences in precision in estimates for each area, the prevalences were also adjusted by empirical Bayes smoothing, i.e. adjusted towards the overall mean. By applying a first-order contiguity rook spatial weight, estimates were also adjusted considering the estimates from neighbouring areas (Bailey and Gatrell, 1995). Presence of spatial autocorrelation was tested using the Moran's $I$ test for global spatial autocorrelation using the same contiguity weight matrix as above (Assuncao and Reis, 1999). To minimize the effect of missing data and prevent "islands" in the maps, postal code areas were first transformed into Thiessen polygons. For presentation purposes, outcomes were later converted back to the original format. In order to classify areas by type of possible spatial correlation, a 


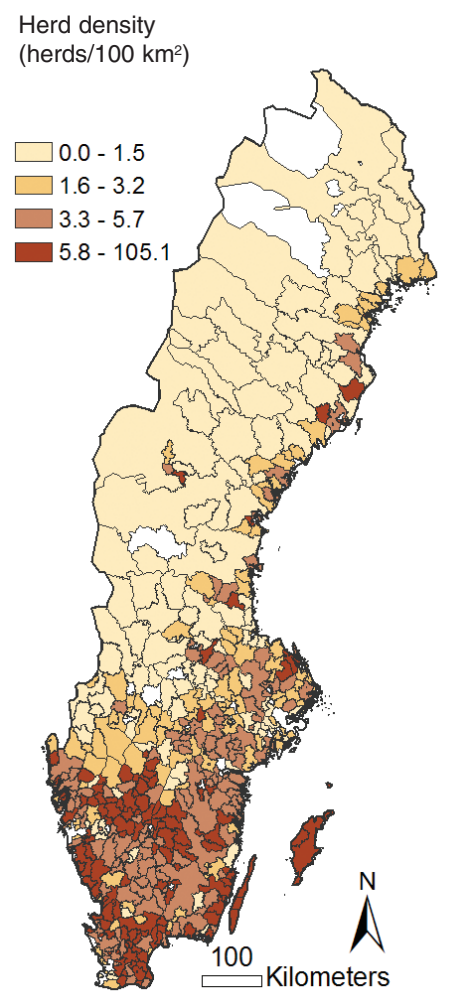

Fig. 1. The population of Swedish dairy herds presented as density by postal code area (2006). () Lantmäteriverket Gävle 2008. Permission number I 2008/1045.

local indicator of spatial association (LISA) test statistic was also applied (Anselin, 1995). Considering the variance instability due to differences in sample size between areas, the calculations were based on the empirical Bayes principle. The number of permutations used was 9,999. All smoothing and testing for spatial associations of area aggregated data was performed using the GeoDa software version 0.9.5-i5 (https://geoda.uiuc.edu/).

Clustering of test-positive herds was also investigated based on location determined by exact coordinates and using the spatial scan statistic ( $\mathrm{M}$. Kulldorff and Information Management Services, Inc. SaTScan ${ }^{\mathrm{TM}}$ version 7.0; www.satscan.org, 2006). The testing was performed using a Bernoulli model (Kulldorff, 1997) where the test-positive herds were considered cases and test-negative herds were considered controls. The maximum cluster sizes tested were $50 \%, 20 \%$ and $10 \%$ of the population at risk and both circular and elliptic cluster shapes were applied. Significance of clusters was tested using Monte Carlo hypothesis testing (9,999 permutations).

Whether or not the study sample was representative of the target population was investigated by calculating the number of sampled herds compared to the total number of dairy herds present in each postal code area. Presence of spatial associations, i.e. a non-random distribution of sampled herds considering the background population, was tested using GeoDa as described above.

Data management, basic statistics and creation of map shape-files were performed using Stata statistical software release 9.2 (Stata Corp., College Station, TX, USA) and ArcView version 9.1 (ESRI Inc., Redlands, CA, USA).

\section{Results}

Herds testing positive for N. caninum ( $\mathrm{n}=247$ ) were found in both the southern, middle and northern parts of the country. However, differences in distribution of positive herds were apparent. The overall prevalence was $8.3 \%$ (95\% CI $=7.3-9.3 \%)$, but a slightly higher prevalence was predominately found in the central parts of Sweden (Fig. 2). This tendency was more obvious when prevalence estimates were adjusted by empirical Bayes smoothing, especially when the spatial weight matrix was applied.

The Moran's $I$ test indicated that spatial autocorrelation was present $(I=0.12, \mathrm{P}=0.004)$ and local spatial correlation tests based on both postal code data and coordinate data identified a high prevalence cluster in the middle of Sweden and a low prevalence cluster in the south of Sweden. However, when applying the LISA test, not all high- or lowprevalence areas were significant at the $\mathrm{P}=0.01$ level (Fig. 3a). One positive and one negative significant cluster were found in most runs of the analyses when using the spatial scan statistic. Although size, shape and exact location of clusters differed 

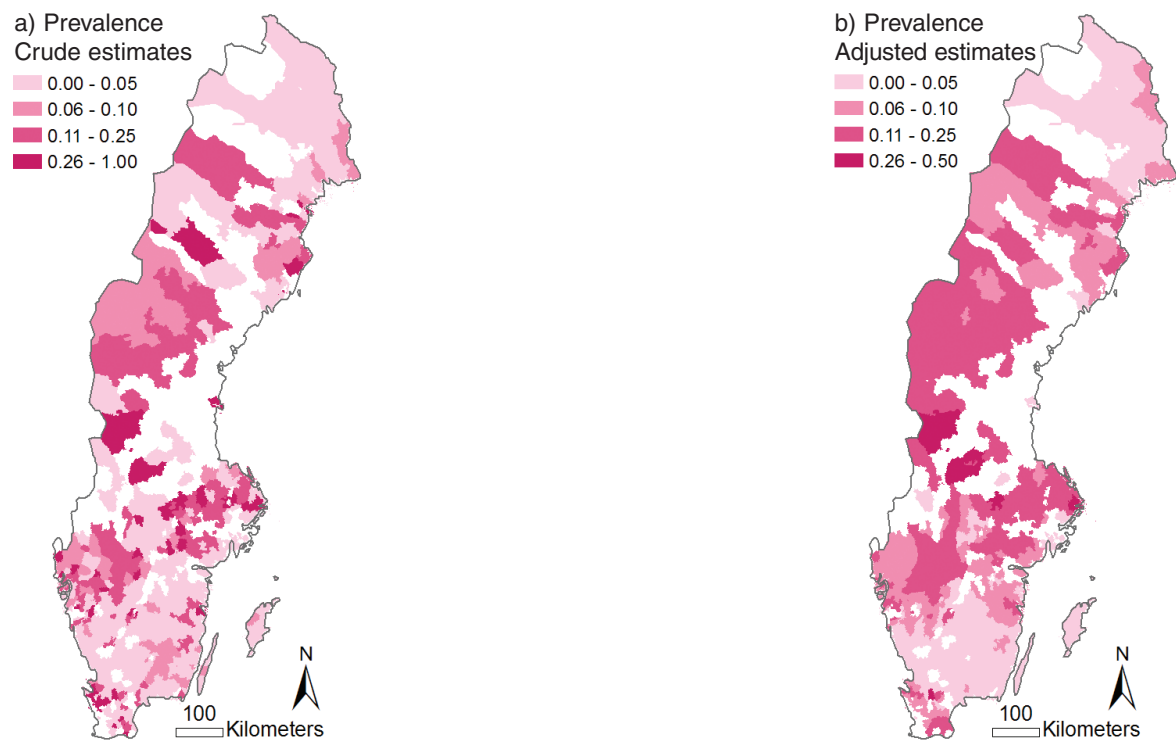

Fig. 2. The prevalence of N. caninum test-positive dairy herds in Sweden by postal code area (total number of sampled herds $=$ 2,944 , number of test-positive herds $=247$ ). The estimates presented are crude (a) or adjusted by empirical Bayes smoothing including applying a spatial weight matrix (b). Information was missing for the white areas. (C) Lantmäteriver-ket Gävle 2008. Permission number I 2008/1045.

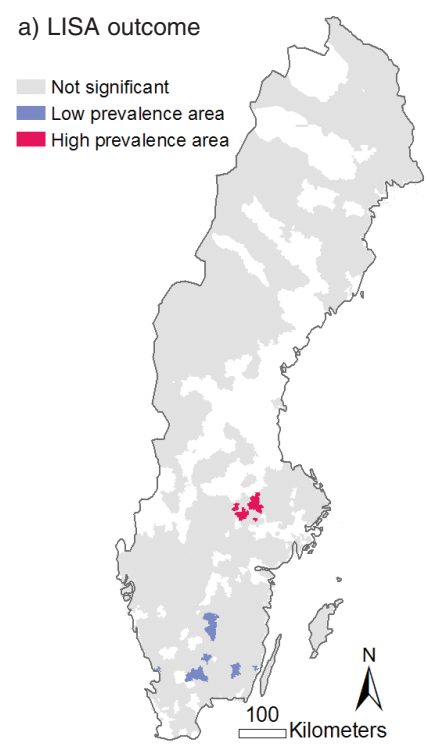

b)

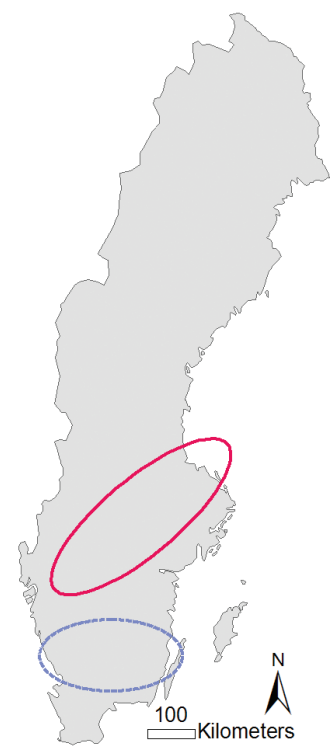

Fig. 3. a) Postal code areas in Sweden identified by a local indicator of spatial association (LISA) test statistic as clusters of high and low prevalence (significance level $\mathrm{P}=0.01$ ) of $N$. caninum test-positive dairy herds in 2002. Areas were transformed into Thiessen polygons before analysis and then converted back to original format for presentation purposes. Information was missing for the white areas. b) Clusters with a significantly higher (red solid line) or lower prevalence (blue dashed line) of N. caninum test-positive dairy herds than expected (Sweden, 2002). Cluster analysis was based on exact coordinates and performed using the spatial scan statistic with a maximum cluster size of $20 \%$ of the population at risk. (C) Lantmäteriverket Gävle 2008. Permission number I 2008/1045. 
depending on settings, all positive clusters were located in the middle parts of Sweden, while all negative clusters were located in the south. The setting with elliptic clusters and a maximum cluster size of $20 \%$ of the population at risk yielded the most informative outcome, which is presented in Figure $3 \mathrm{~b}$. The identified low-risk cluster in the south included 486 herds and had 12 observed cases versus 42 expected (relative risk $(\mathrm{RR})=0.25, \mathrm{P}=$ 0.0002 ) while the high-risk cluster in the middle of Sweden included 490 herds and had 80 observed cases versus 42 expected ( $\mathrm{RR}=2.43, \mathrm{P}=0.0002)$.

Some postal code areas $(\mathrm{n}=125$, i.e. $31 \%)$ were

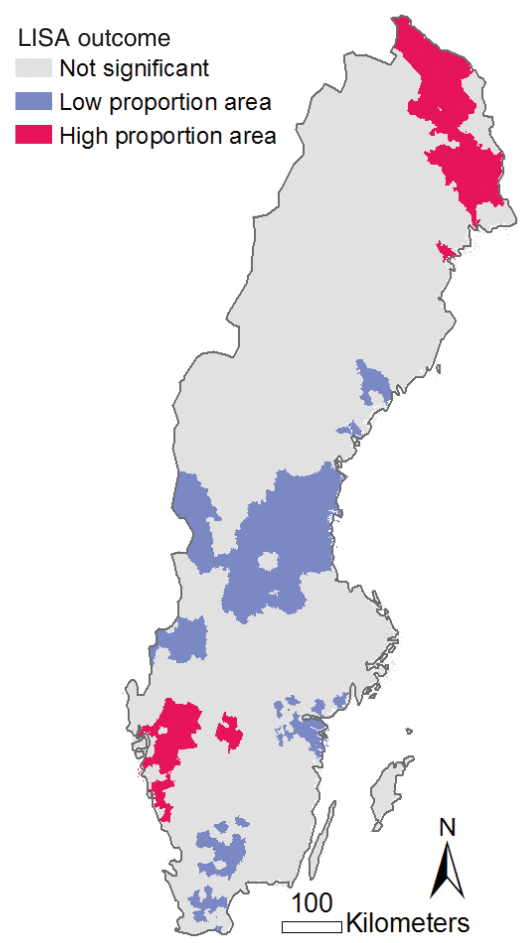

Fig. 4. Regions that were underrepresented or overrepresented in the present study sample of dairy herds that was used to investigate the spatial distribution of $N$. caninum test-positive herds in Sweden. The study sample $(\mathrm{n}=2,944)$ was selected in 2002 and was compared to the background population of dairy herds in Sweden 2006 ( $\mathrm{n}=8,288)$. Geographical representativeness was investigated by calculating a local indicator of spatial association (LISA) test statistic, significance level $\mathrm{P}=0.01$. (C) Lantmäteriverket Gävle 2008. Permission number I $2008 / 1045$. missing in the selected study sample. In particular, the central-east areas and some southern areas were underrepresented. On the other hand, in some of the regions in the north and south-west, a very high proportion of existing herds was sampled (Fig. 4). These differences in sampling density were significant when investigated using cluster analysis $(\mathrm{P}<0.01$ in the LISA test statistic).

\section{Discussion}

In this study, both visual inspection and exploratory spatial analyses of data indicated that there is a higher prevalence of $N$. caninum infected herds in the middle parts of Sweden and a lower prevalence in the south. The overall prevalence of infected herds was lower than previous estimates, which on the other hand were limited to the south to middle regions. Because the previous estimate of diagnostic sensitivity of the test used was based on a small number of herds (and the confidence interval consequently was very wide) it is difficult to estimate the true prevalence of infection. Still, it is likely that the proportion of herds with N. caninum infected individuals is higher than the estimated $8.3 \%$.

Although the geographical distribution of herds testing positively roughly coincided with the distribution of known case herds (data not shown), it was not expected that the south of the country would have a significantly lower prevalence of $N$. caninum. Other studies have shown that, with a few exceptions, the prevalence of $N$. caninum is higher in the southern parts of Europe compared to the northern parts (Hemphill and Gottstein, 2000; Bartels et al., 2006). There are also indications that within regions, the prevalence is higher in areas with higher temperatures in the spring and summer period (Schares et al., 2003; Rinaldi et al., 2005). In addition, herd sizes are larger and the herd density is higher in the south of Sweden than in other parts of the country. These demographic features are often considered risk factors for a higher prevalence/incidence of infectious diseases. 
The few studies that have investigated possible effects on the spatial distribution of $N$. caninum infection in cattle have shown a significant positive correlation with the density of dogs and the vegetation profile (Schares et al., 2003; Rinaldi et al., 2005). Further analyses of the presented data in order to investigate the effect of such covariates will require additional data from several different sources of information. One factor that could have a possible influence on the results obtained is the distribution of the Scandinavian wolf population. Although few in numbers, breeding pairs of wolves are reported to be located in the middle parts of Sweden (Aronson and Svensson, 2007) which, in the present study, has been identified as a high N. caninum prevalence area.

The date of origin differed for some of the information used in this study. As N. caninum causes a chronic infection which by nature is somewhat slow in spreading, these differences are probably of minor significance. It could be expected that data regarding more of the herds in the study sample would have been available if an earlier version of the database could have been obtained. On the other hand, just a few years ago, information retrieval based on specific coordinates was not as developed as it is today.

Possible edge effects were not considered in this analysis. However, since the major parts of Sweden's borders are either coastline or very sparsely-populated mountain areas, it is likely that outcomes from the cluster analyses are more or less uninfluenced by edge effects and that estimates from spatial smoothing were only affected to a limited extent.

One objective of this study was to investigate the geographical distribution of the study sample. It was found that, compared to the background population, a disproportionally large number of samples had been selected from the very north and the middle-west parts of the country. On the other hand, many postal code areas in the central-east areas, where several dairy herds are located, were not at all represented in the study sample. Also, too few samples had been selected from areas in the south. This adds uncertainty to the interpretation of some of the results. Close proximity to the high-prevalence middle area of the country could indicate that the central-east region also has a relatively high number of infected herds. This region was indeed included in, or was close to, some of the significant clusters of cases identified by cluster analysis based on exact coordinates. There is a fairly large proportion of previously known case herds in this area, which supports the credibility of this finding.

Although data are missing for some regions, it can be concluded that there are geographical differences in the distribution of $N$. caninum infected dairy herds in Sweden and that the overall tendency is that prevalence is higher in the middle parts of the country and lower in the southern parts. This information substantially adds to the current knowledge of $N$. caninum infection in Swedish cattle and can be used in further epidemiological studies of this parasite.

\section{Acknowledgements}

The skilful technical assistance provided by Katarina Näslund was greatly appreciated. We also thank GIS coordinator Göran Adelsköld of the Swedish University of Agricultural Sciences for advice and help with software/maps. Jenny Frössling was financially supported by the Swedish Emergency Management Agency.

\section{References}

Anonymous, 2001. PC-Atlasen GIS, version 2.0. Sveriges Nationalatlas, KartCentrum, Vällingby, Sweden.

Anonymous, 2008. Surveillance and control programs domestic and wild animals in Sweden 2007. SVAs rapportserie 4, ISSN 1654-7098. National Veterinary Institute, Uppsala, Sweden. Available at: http://www.sva.se/sv/navigera/tjanster_produkter/Trycksaker (Accessed: August 2008).

Anselin L, 1995. Local indicators of spatial association LISA. Geogr Anal 27, 93-115.

Aronson A, Svensson L, 2007. Varg i Sverige vintern 2006/2007. [Wolves in Sweden.] Status report (in Swedish), Viltskadecenter, Grimsö Wildlife Research Station, Sweden. 
Assuncao RM, Reis EA, 1999. A new proposal to adjust Moran's I for population density. Stat Med 18, 2147-2162. Bailey TC, Gatrell AC, 1995. Further methods for area data. In: Interactive Spatial Analysis, Longman Group Limited, Burnt Mill, Harlow, Essex, pp. 298-328.

Barling KS, Sherman M, Peterson MJ, Thompson JA, McNeill JW, Craig TM, Adams LG, 2000. Spatial associations among density of cattle, abundance of wild canids, and seroprevalence to Neospora caninum in a population of beef calves. J Am Vet Med Assoc 217, 1361-1365.

Bartels CJ, Arnaiz-Seco JI, Ruiz-Santa-Quitera A, Björkman C, Frössling J, von Blumröder D, Conraths FJ, Schares G, van Maanen C, Wouda W, Ortega-Mora LM, 2006. Supranational comparison of Neospora caninum seroprevalences in cattle in Germany, The Netherlands, Spain and Sweden. Vet Parasitol 137, 17-27.

Björkman C, Alenius S, Emanuelson U, Uggla A, 2000. Neospora caninum and bovine virus diarrhoea virus infections in Swedish dairy cows in relation to abortion. Vet J 159, 201-206.

Björkman C, Holmdahl OJ, Uggla A, 1997. An indirect enzyme-linked immunoassay (ELISA) for demonstration of antibodies to Neospora caninum in serum and milk of cattle. Vet Parasitol 68, 251-260.

Björkman C, Lundén A, Uggla A, 1994. Prevalence of antibodies to Neospora caninum and Toxoplasma gondii in Swedish dogs. Acta Vet Scand 35, 445-447.

Dubey JP, Schares G, Ortega-Mora LM, 2007. Epidemiology and control of neosporosis and Neospora caninum. Clin
Microbiol Rev 20, 323-367.

Frössling J, Bonnett B, Lindberg A, Björkman C, 2003. Validation of a Neospora caninum iscom ELISA without a gold standard. Prev Vet Med 57, 141-153.

Frössling J, Lindberg A, Björkman C, 2006. Evaluation of an iscom ELISA used for detection of antibodies to Neospora caninum in bulk milk. Prev Vet Med 74, 120-129.

Gondim LF, McAllister MM, Pitt WC, Zemlicka DE, 2004. Coyotes (Canis latrans) are definitive hosts of Neospora caninum. Int J Parasitol 34, 159-161.

Hemphill A, Gottstein B, 2000. A European perspective on Neospora caninum. Int J Parasitol 30, 877-924.

Kulldorff M, 1997. A spatial scan statistic. Commun Stat Theory Methods 26, 1481-1496.

McAllister MM, Dubey JP, Lindsay DS, Jolley WR, Wills RA, McGuire AM, 1998. Dogs are definitive hosts of Neospora caninum. Int J Parasitol 28, 1473-1478.

Rinaldi L, Fusco G, Musella V, Veneziano V, Guarino A, Taddei R, Cringoli G, 2005. Neospora caninum in pastured cattle: determination of climatic, environmental, farm management and individual animal risk factors using remote sensing and geographical information systems. Vet Parasitol 128, 219-230.

Schares G, Barwald A, Staubach C, Ziller M, Kloss D, Wurm R, Rauser M, Labohm R, Drager K, Fasen W, Hess RG, Conraths FJ, 2003. Regional distribution of bovine Neospora caninum infection in the German state of Rhineland-Palatinate modelled by logistic regression. Int J Parasitol 33, 1631-1640. 